\title{
MORBIMORTALIDADE RELACIONADA À TÉCNICA DE ANASTOMOSE PANCREÁTICA(DUCTO-MUCOSA X TELESCOPAGEM) APÓS CIRURGIA DE WHIPPLE
}

\author{
MORBIMORTALITY RELATED TO PANCREATIC ANASTOMOSIS TECHNIQUE \\ (DUCT-TO-MUCOSA VS. TELESCOPIC) AFTER WHIPPLE'S SURGERY
}

\author{
Jorge Mali Junior ${ }^{1}$; Gustavo Stoduto Carvalho ${ }^{1}$; Gustavo Pierro ${ }^{1}$; \\ Marciano Anghinoni ${ }^{1}$; Jurandir de Almeida Dias, ACBC-RJ ${ }^{2}$; Rafael Albagli,TCBC-RJ ${ }^{3}$
}

\begin{abstract}
RESUMO: Objetivos: A gastroduodenopancreatectomia ( GDP) é atualmente a única forma de tratamento segura e eficaz para pacientes selecionados com doenças benignas e malignas do pâncreas e da região periampular. Entre as complicações pós-operatórias, a fístula pancreática continua sendo a mais importante, com uma incidência que varia de 5 a $25 \%$ nas grandes séries. Os objetivos deste trabalho são os de avaliar a morbimortalidade relacionada a duas técnicas de anastomoses pancreatojejunais (ducto-mucosa $\mathrm{X}$ telescopagem), e comparar seus resultados. Método: Foram analisados retrospectivamente 64 pacientes submetidos à GDP, no Serviço de Cirurgia Abdômino-Pélvica, do INCA, no período de 1987 a 2002. Destes doentes, 42 foram submetidos à anastomose tipo ducto-mucosa e 22 à telescopagem. A análise estatística foi realizada através do teste de Fischer. Resultados: A taxa de fístula pancreática no grupo ducto-mucosa foi de $12 \%$ e no telescopagem foi $36 \%$. Esta diferença percentual se mostrou estatisticamente significativa $(\mathrm{p}=0,02)$. A mortalidade operatória relacionada à fístula pancreática foi de $2,4 \%$ para o grupo ducto-mucosa e $4,5 \%$ para o telescopagem, com nível de significância estatística > 5\%. Conclusões: A técnica de anastomose pancreatojejunal tipo ducto-mucosa é associada a menores índices de fístula pancreática em relação a técnica de telescopagem, enquanto que a mortalidade operatória relacionada a fístula não mostrou diferença estatística entre os dois grupos estudados (Rev. Col. Bras. Cir. 2005; 32(2): 74-77).
\end{abstract}

Descritores: Anastomose cirúrgica; Pâncreas; Cirurgia

\section{INTRODUÇÃO}

A gastroduodenopancreatectomia (GDP) é atualmente a única forma de tratamento segura e eficaz para pacientes selecionados com patologias benignas e malignas do pâncreas e da região periampular. No entanto, desde que Whipple publicou três casos de ressecção pancreaticoduodenal em 1935, esta cirurgia é originalmente associada a alta morbimortalidade ${ }^{1}$.

Com os recentes avanços da cirurgia pancreática e dos cuidados pós-operatórios, a GDP tem hoje uma baixa taxa de mortalidade $(<5 \%)^{2-5}$. Entretanto, esta operação é ainda associada a uma considerável morbidade pós-operatória, atingindo até $50 \%$ dos casos em alguns centros especializados ${ }^{2}$.

Entre as complicações, a fístula pancreática continua sendo a mais importante, com uma incidência que varia de 5 a $25 \%$ nas grandes séries ${ }^{3-6}$. Em atenção a este, que é hoje, o maior problema das ressecções pancreticoduodenais, nosso estudo objetivou avaliar a morbimortalidade relacionada a duas técnicas de anastomoses pancreatojejunais (ductomucosa X telescopagem), e comparar seus resultados.

\section{MÉTODO}

Foram analisados retrospectivamente 64 pacientes submetidos à GDP, no Serviço de Cirurgia Abdômino-Pélvica, do INCA, no período de 1987 a 2002.
Todas as cirurgias, independente da técnica utilizada, foram realizadas pela mesma equipe de cirurgiões, com experiência semelhante. Além disso, as duas técnicas em questão, não sofreram qualquer influência temporal, sendo realizadas por todo o período do estudo.

Todos os pacientes foram submetidos à GDP associada à linfadenectomia standard, com reconstrução do trânsito alimentar e bílio-pancreático em alça jejunal única. A gastrojejunoanastomose e a hepaticojejunoanastomose foram término-laterais, com plano único de sutura, extramucoso, com fio de prolene 3.0.

Os dois grupos eram homogêneos quanto as características demográficas e localização tumoral (Tabela 1). Quanto ao estadiamento clínico, segundo o sistema TNM empregado pela união internacional contra o câncer (UICC), os pacientes de ambos os grupos tinham doença ressecável, sem metástase à distância.

Quanto à anastomose pancreática, realizamos duas técnicas diferentes. A pancreatojejunoanastomose tipo ducto-mucosa consiste em secção de $5 \mathrm{~cm}$ da camada seromuscular da alça jejunal, expondo sua mucosa. Sutura extramucosa da parede posterior do jejuno com o parênquima pancreático, com pontos separados de prolene 5.0. Diérese de cerca de $3 \mathrm{~mm}$ na mucosa jejunal, suturando esta com o ducto de Wirsung através de quatro pontos cardeais de prolene 5.0. Sutura extramucosa da parede anterior jejunal

1. Médico Residente de Cirurgia Oncológica do INCA.

2. Chefe do Serviço de Cirurgia Abdômino-Pélvica do INCA.

3. Coordenador do Grupo de Pâncreas do Serviço de Cirurgia Abdômino-Pélvica do INCA; Mestrando do Curso de Pós-Graduação em Cirurgia da UFRJ.

Recebido em 24/11/2004

Aceito para publicação em 04/01/2005

Trabalho realizado na Seção de Cirurgia Abdômino-Pélvica do Instituto Nacional Câncer (RJ) - Ministério da Saúde. 
Tabela 1 - Características demográficas e topografia tumoral dos grupos analisados.

\begin{tabular}{lll}
\hline & Grupo Ducto-mucosa $(\mathbf{n = 4 2})$ & Grupo telescopagem $(\mathbf{n = 2 2})$ \\
\hline Idade & $18-80(57$ anos $)$ & $17-72(55$ anos $)$ \\
Sexo & Masculino $=21(50 \%)$ & Masculino $=9(41 \%)$ \\
& Feminino $=21(50 \%)$ & Feminino $=13(59 \%)$ \\
Tumores Periampulares & Cabeça do pâncreas $=16(38 \%)$ & Papila=10 $(45 \%)$ \\
& Papila $=15(36 \%)$ & Cabeça do pâncreas $=8(36 \%)$ \\
& Duodeno $=3(7 \%)$ & Duodeno=1 $(5 \%)$ \\
Tumor Não-Periampulares & Cólon $=4(9 \%)$ & Cólon $=3(14 \%)$ \\
& Estômago $=2(5 \%)$ & \\
& Intestino Delgado=2 $(5 \%)$ & \\
\hline
\end{tabular}

com o parênquima pancreático, com pontos separados de prolene 5.0 (Figura 1).

A técnica de telescopagem, era realizada de forma término-terminal, em dois planos de sutura, com pontos separados de vicryl 4.0, sendo um plano total envolvendo a parede jejunal e a borda pancreática, e o outro entre a seromuscular do jejuno e a cápsula pancreática, invaginando assim o coto pancreático e o ducto principal para o lúmen intestinal por 2 a $4 \mathrm{~cm}$ (Figura 2).

Não foram utilizados cateteres transanastomóticos em nenhuma das técnicas empregadas neste estudo. A cavidade abdominal era drenada com dois drenos tubulares em sistema fechado, sentinela as anastomoses gástrica, biliar e pancreática, exteriorizados nos hipocôndrio direito e esquerdo. No pós-operatório precoce era instituída de rotina a dieta enteral.

Foram considerados portadores de fístula pancreática aqueles pacientes cuja concentração de amilase na secreção do dreno abdominal fechado sentinela a anastomose pancreática, tivesse o valor de três vezes a amilase sérica, por volta do $7^{\circ}$ dia pós-operatório. Estabeleceu-se como óbito pósoperatório aquele ocorrido até o $30^{\circ}$ dia após a cirurgia.

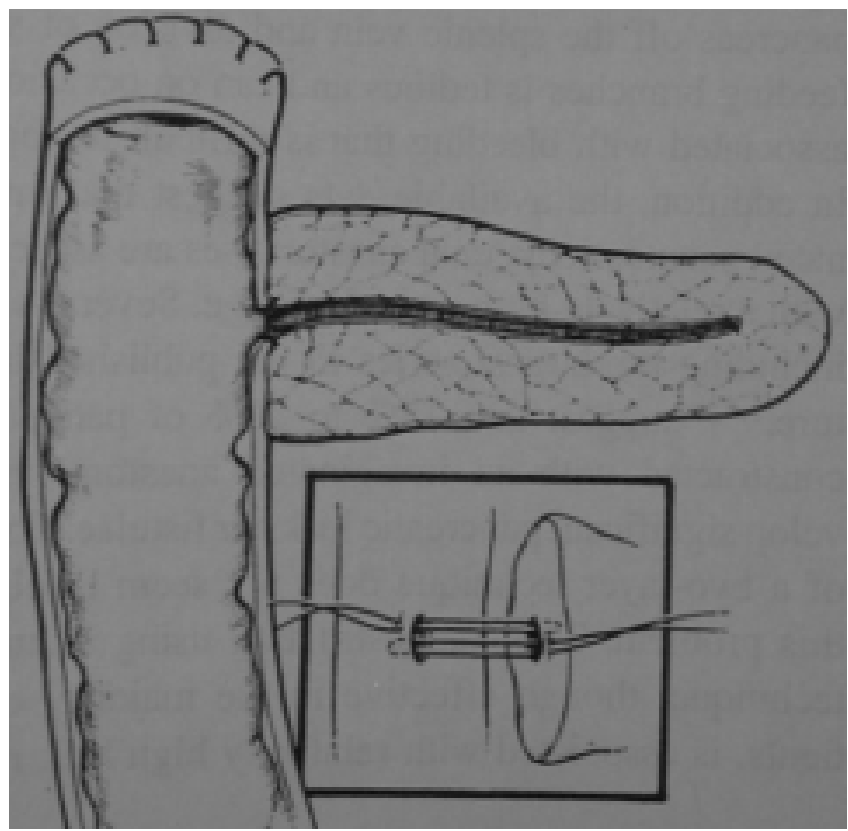

Figura 1 - Anastomose pancreatojunal término-lateral (ducto-mucosa sem cateter transanatomótico).
A análise estatística foi realizada empregando o teste exato de Fisher para a comparação entre os dois tipos de técnica e a taxa de morbimortalidade.

\section{RESULTADOS}

Dos 64 doentes analisados, 34 eram do sexo feminino e 30 do masculino, com mediana de idade de 54 anos (18 80). As indicações foram tumores da ampola de Vater em 25 pacientes, neoplasia maligna do pâncreas em 24 pacientes e do duodeno em quatro doentes. Os tumores primários nãoperiampulares se fizeram presente em 11 doentes, sendo sete casos com origem no cólon, dois casos no estômago e dois no intestino delgado.

Histologicamente todos os casos de tumores periampulares eram adenocarcinomas, enquanto entre os nãoperiampulares haviam oito casos de adenocarcinoma (câncer de cólon $=$ sete e câncer gástrico $=u m$ ) três de sarcoma (intestino delgado $=$ dois e estômago $=$ um).

Quanto à anastomose pancreática, em 42 casos foi empregada a anastomose tipo ducto-mucosa (tumor de cabeça de pâncreas $=16$; tumor de papila $=15$; tumor nãoperiampular $=$ oito e tumor de duodeno =três) e nos 22 restantes a telescopagem (tumor de papila $=10$; tumor de pâncreas=oito; tumor de duodeno=um e tumor não periampular $=$ três).

A mediana do tempo operatório e de internação para o grupo ducto-mucosa (42 pacientes) foram respectivamente, 417 minutos $(180$ - 660) e 15 dias (2 - 57). O grupo telescopagem teve uma mediana de tempo cirúrgico de 420 minutos $(300$ - 705) e o tempo de internação hospitalar variou de dois a 60 dias, com mediana de 15,5 dias ( não houve diferença estatisticamente significante entre os dois grupos).

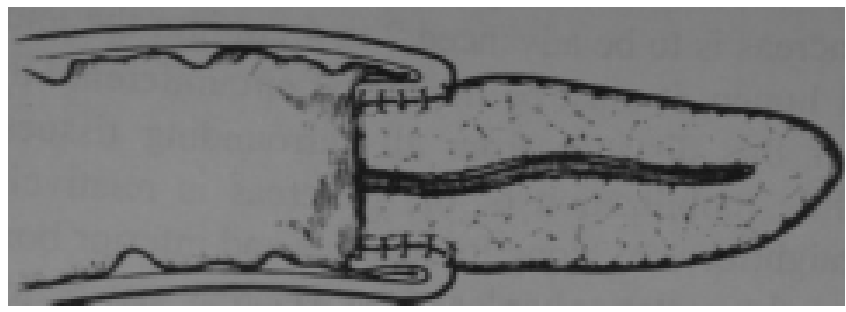

Figura 2 - Anastomose pancreatojunal término-terminal (telescopagem). 
Dos 64 pacientes, 22 foram submetidos à transfusão sangüínea, 11 do grupo telescopagem $(50 \%)$ e 11 do ductomucosa (26\%). A mediana de volume sangüíneo transfundido foi de $900 \mathrm{ml}(300$ - 1500) para o grupo ducto-mucosa e $800 \mathrm{ml}$ $(300-1880)$ para os pacientes submetidos a anastomose tipo telescopagem.

A taxa global de fístula pancreática foi de $20 \%$ (13 casos), sendo que no grupo ducto-mucosa foi de $12 \%$ (cinco casos) e no telescopagem foi $36 \%$ (oito doentes). Esta diferença percentual se mostrou estatisticamente significativa $(p=0,02)$.

A mortalidade operatória relacionada a fístula pancreática foi de $2,4 \%$ (um caso) para o grupo ducto-mucosa e $4,5 \%$ (um doente) para o telescopagem, com nível de significância estatística $>5 \%(\mathrm{p}=0,46)$.

\section{DISCUSSÃO}

O tratamento cirúrgico dos tumores periampulares teve início há várias décadas ${ }^{7-9}$. No entanto, somente em 1935 após três duodenopancreatectomias realizadas por Whipple ${ }^{7}$ que o procedimento ficou mundialmente conhecido.

Durante a década de 60 e 70, muitos autores sugeriam que a cirurgia fosse abandonada devido a seus índices alarmantes de morbimortalidade ${ }^{10,11}$. Felizmente, com os avanços tecnológicos, no que diz respeito à anestesia, materiais cirúrgicos, manejo pós-operatório, nutrição enteral e parenteral, atualmente a cirurgia de Whipple é realizada com uma mortalidade operatória menor que 5\%. Apesar disso, este procedimento continua tendo uma morbidade alta, atingindo um índice de até $50 \%$ em centros de excelência em cirurgia pancreática ${ }^{2}$.

A fístula pancreática é sua complicação mais temida, com $80 \%$ dos óbitos decorrentes dela ${ }^{6}$. Um recente estudo relatou que a incidência de fístula pancreática após ressecções duodenopancreáticas não tem mudado desde a década passada, situando-se entre 5 e $25 \%{ }^{6,12}$. Por outro lado, a opção de não se realizar reconstrução pancreatoentérica, ocluindo o ducto pancreático sem anastomose é associado a índices proibitivos de fístula pancreática e insuficiência endócrina.

Cattell ${ }^{13}$, em 1943, estabeleceu que a anastomose pancreatoentérica era indispensável, eliminando definitivamente a idéia de ligadura do ducto pancreático principal. Logo em seguida, Whipple também introduziu a pancreatojejunoanastomose e concluiu a reconstrução em um estágio, dando o epônimo a GDP que temos hoje de cirurgia de Whipple.

As anastomoses pancreatoentéricas empregadas atualmente são uma combinação de várias técnicas cirúrgicas, a depender do local e direção da anastomose, e método de sutura, entre outros ${ }^{14}$. Geralmente, entretanto, os tipos de anastomoses podem ser divididas em término-terminais (telescopagem) e término-laterais (ducto-mucosa). A pancreatojejunoanastomose término-terminal proposta por Child ${ }^{15}$, é o método que introduz o coto pancreático no lúmen jejunal e com isso o suco pancreático é drenado totalmente no trato intestinal. As desvantagens desse método são o tempo cirúrgico maior, sangramento do coto pancreático freqüente e, uma vez rompida a anastomose, todo o conteúdo do trato intestinal entra em contato com a cavidade abdominal, causando sérios problemas. Já a anastomose término-lateral introduzida por Cattell e Whipple, apesar do tempo operatório ser semelhante ao tipo termino-terminal, o sangramento do coto pancreático é improvável devido a sutura da parede anterior e posterior deste com a alça intestinal. Um problema desta anastomose é o espaço morto que se faz entre o coto do pâncreas e a parede intestinal que pode acarretar pancreatite e deiscência.

Muitos fatores, entre outros citados acima, podem influenciar a ocorrência de deiscência da anastomose pancreatoentérica, tais como experiência do cirurgião, método de reconstrução, técnica anastomótica, tipo e qualidade do ducto pancreático remanescente, uso de cateteres transanastomóticos e drenos abdominais.

Várias técnicas cirúrgicas e modificações tem sido propostas durante décadas para reconstrução pancreática após GDP, mas a despeito disso a taxa de fístula pancreática continua alta ${ }^{16,17}$. Pancreatogastroanastomose tem sido uma alternativa mais comumente usada em relação à pancreatojejunoanastomose, mas apesar de taxas de morbimortalidade semelhantes, o sangramento anastomótico tem limitado o uso da anastomose gastropancreática ${ }^{18,19}$.

Existem poucos trabalhos na literatura comparando especificamente morbimortalidade operatória relacionada ao tipo de anastomose pancreática após GDP. De acordo com um recente estudo prospectivo randomizado, a taxa de fístula pancreática seguido de anastomose ducto-mucosa foi de $4 \%$ e após anastomose termino-terminal tipo telescopagem foi de $15 \%$, porém sem diferença estatística $(\mathrm{p}=0,09)$. A mortalidade operatória para o grupo ducto-mucosa foi $6 \%$, enquanto para o grupo telescopagem foi $9 \%{ }^{19}$.

Matsumoto et al ${ }^{20}$ relataram que a taxa de fístula pancreática é influenciada pela técnica anastomótica, e que a técnica ducto-mucosa término-lateral tem uma incidência de fístula menor em relação a outros tipos de anastomose.

$\mathrm{O}$ uso de cateteres transanastomóticos para prevenir obstrução ductal e acelerar a cicatrização da anastomose pancreática é uma tática cirúrgica utilizada por muitos cirurgiões, com bons resultados, apesar de sua efetividade nunca ter sido comprovada ${ }^{21}$. Acreditamos que o uso, de rotina, de tubos através da anastomose pancreática seja dispensável porque o processo de cicatrização entre a mucosa jejunal e o ducto pancreático se faz no pós-operatório precoce, além do que o paciente requer cuidados mais complexos quanto maior o número de cateteres exteriorizados. Na nossa instituição, o uso de stent está atrelado a duas situações: preferência do cirurgião e ducto pancreático principal fino.

O uso de drenos ainda é controverso ${ }^{22,23}$. A nossa conduta é drenar rotineiramente as anastomoses biliar e pancreática através de dois drenos fechados exteriorizados nos hipocôndrios direito e esquerdo. A drenagem de rotina da anatomose pancreática pode ainda prevenir a formação de abscessos intracavitários nos pacientes com fístulas de baixo débito.

O papel do octreotide na prevenção da fístula pancreática permanece controverso ${ }^{19}$. Neste estudo os pacientes não receberam octreotide. 
Baseados neste estudo concluímos que a técnica de anastomose pancreatojejunal tipo ducto-mucosa, sem o uso de cateteres transanastomóticos, é associada a menores índices de fístula pancreática em relação a técnica de telescopagem, enquanto que a mortalidade operatória relacionada à fístula não mostrou diferença estatística entre os dois grupos estudados. É claro que são necessários novos estudos, com maior amostragem e com delineamento prospectivo, para definir a influência da técnica da anastomose pancreática na morbimortalidade após GDP, cirurgia esta que continua sendo um desafio para o cirurgião pancreático.

\begin{abstract}
Background: Pancreaticoduodenectomy has been used as a safe and appropriate surgical option in selected patients with malignant and benign disorders of the pancreas and periampullary region. Among the complications of the pancreatoduodenectomy, pancreatic anastomosis failure is the most important and sometimes fatal complication. According to recent reports, the incidence of leakage of pancreatic anastomosis after PD is high (between $5 \%$ and $25 \%$ ). Our objectives were to analize the morbidity and mortality rates related to pancreatic anastomosis technique (duct-to-mucosa VS. telescopic) and to compare the results. Methods: From January 1987 to December 2002, 64 patients underwent pancreaticoduodenal resection at Brazilian National Cancer Institute. Data were recorded retrospectively on all patients. Forty-two patients underwent pancreatojejunostomy duct-to-mucosa and the telescopic technique was performed in 22 patients. Statistical analyses were performed using the Fischer's test. Results: The pancreatic fistula rate after pancreatojejunostomy duct-to-mucosa and telescopic technique was $12 \%$ and $36 \%$, respectively $(p=$ 0.02). The operative mortality rate after duct-to-mucosa technique was $12 \%$ and $36 \%$ after pancreatojejunostomy telescopic $(p=0.4)$. Conclusions: The leakage rate was significantly lower in patients underwent to pancreatojejunostomy duct-to-mucosa, however an operative mortality rate was not significantly different between the two groups.
\end{abstract}

Key-words: Anastomosis, surgical; Pancreas; Surgery.

\section{REFERÊNCIAS}

1. Whipple AO, Parsons WB, Mullins CR - Treatment of carcinoma of the ampulla of vater. Ann Surg. 1935, 102:763-779.

2. Yeo CJ, Cameron JL, Sohn TA, et al. - Six hundred fifty consecutive pancreaticoduodenectomies in the 1990s: pathology, complications, and outcomes. Ann Surg. 1997, 226(3):248-260

3. Yeo CJ, Cameron JL, Lillemoe KD, et al. - Does prophylactic octreotide decrease the rates of pancreatic fistula and other complications after pancreaticoduodenectomy? Results of a prospective randomized placebo-controlled trial. Ann Surg. 2000, 232(3):419-429.

4. Suzuki Y, Fujino Y, Tanioka Y, et al. - Selection of pancreaticojejunostomy techniques according to pancreatic texture and duct size. Arch Surg. 2002, 137(9): 1044-1048.

5. Gouma DJ, Van Geenen RC, Van Gulik TM, et al. - Rates of complications and death after pancreaticoduodenectomy: risk factors and the impact of hospital volume. Ann Surg. 2000, 232(6):786-795.

6. van Berge Henegouwen MI, de Wit LT, Van Gulik TM, et al. Incidence, risk factors, and treatment of pancreatic leakage after pancreaticoduodenectomy: drainage versus resection of the pancreatic remnant. J Am Coll Surg. 1997, 185(1):18-24.

7. Whipple AO - A historical sketch of the pancreas. In Howard JM, Jordan GL Jr (eds) - Surgical disease of the pancreas. JB Lippincott, Philadelphia, 1960. pp.1-8.

8. Kausch W-Das carcinoma der papilla duodeni und seine radikale entfeinung. Beitr Z Clin Chir. 1912, 78:439-486.

9. Hirschel G - Die resecktion des duodenum mit der papilla wegen karzinoms. Munch Med Wsch. 1914, 61:1728-1729.

10. Crile $\mathrm{G}$ - The advantages of bypass operations over radical pancreatoduodenectomy in the treatment of pancreatic carcinoma. Surg Gynecol Obstet. 1970, 130(6):1049-1053.

11. Shapiro TM - Adenocarcinoma of the pancreas: a statistical analysisof biliary bypass vs. Whipple resection in good risk patients. Ann Surg. 1975, 182:715-721.

12. Balcom JH, Rattner DW, Warshaw AL, et al. - Ten-year experience with 773 pancreatic resections: changing indications, older patients, and decreasing length of hospitalization. Arch Surg. 2001, 136(4):391-398.

13. Cattell RB - Resection of the pancreas, discussion of special problems. Surg Clin North Am. 1943, 23:753-766.

14. Kakita A, Yoshida M, Takahashi T - History of pancreaticojejunostomy in pancreaticoduodenectomy: development of a more reliable anastomosis technique. J Hepatobiliary Pancreat Surg. 2001, 8(3):230-237.

15. Child CG - Carcinoma of duodenum: one-stage radical pancreaticoduodenectomy preserving the external pancreatic secretion, case report. Ann Surg. 1941, 118:838-842.

16. Matsusue S, Takeda H, Nakamura Y, et al. - A prospective analysis of the factors influencing pancreaticojejunostomy performed using a single method, in 100 consecutive pancreaticoduodenectomies. Surg Today. 1998, 28(7):719-726.

17. Martin FM, Rossi RL, Munson JL, et al. - Management of pancreatic fistulas. Arch Surg. 1989, 124(5):571-573.

18. Yeo CJ, Cameron JL, Maher MM, et al. - A prospective randomized trial of pancreaticogastrostomy versus pancreaticojejunostomy after pancreaticoduodenectomy. Ann Surg. 1995, 222(4):580-592

19. Stojadinovic A, Brooks A, Hoos A, et al. - An evidence-based approach to the surgical management of resectable pancreatic adenocarcinoma. J Am Coll Surg. 2003, 196(6):954-964.

20. Matsumoto Y, Fujii H, Miura K, et al . - Successful pancreatojejunal anastomosis for pancreatoduodenectomy. Surg Gynecol Obstet. 1992, 175(6):555-562.

21. Biehl T, Traverso LW - Is stenting necessary for a successful pancreatic anastomosis? Am J Surg. 1992, 163(5):530-532.

22. Conlon KC, Labow D, Leung D, et al. - Prospective randomized clinical trial of the value of intraperitoneal drainage after pancreatic resection. Ann Surg. 2001, 234(4):487-493.

23. Z'graggen $\mathrm{K}$, Uhl W, Friess $\mathrm{H}$, et al. - How to do a safe pancreatic anastomosis. J Hepatobiliary Pancreat Surg. 2002, 9(6):733-737.

Endereço para correspondência:

Rafael Albagli

Rua Muniz Barreto, 396 - apto. 1002

22251-090 - Botafogo - Rio de Janeiro - RJ 